\title{
Spread through air spaces in lung neuroendocrine tumor
}

\author{
Yuka Kozuma $^{1}$, Gouji Toyokawa ${ }^{1}$, Yuichi Yamada ${ }^{2}$, Fumihiro Shoji ${ }^{1}$, Koji Yamazaki ${ }^{1}$, Yoshinao Oda ${ }^{2}$, \\ Sadanori Takeo ${ }^{1}$
}

${ }^{1}$ Department of Thoracic Surgery, Clinical Research Institute, National Hospital Organization, Kyushu Medical Center, Fukuoka, Japan; ${ }^{2}$ Department of Anatomic Pathology, Graduate School of Medical Sciences, Kyushu University, Fukuoka, Japan Correspondence to: Gouji Toyokawa, MD, PhD. Department of Thoracic Surgery, Clinical Research Institute, National Hospital Organization, Kyushu Medical Center, 1-8-1 Jigyohama, Chuo-ku, Fukuoka 810-8563, Japan. Email: gouji10yokawa@gmail.com.

Provenance: This is an invited article commissioned by the Academic Editor Dr. Chenyang Dai (Department of Thoracic Surgery, Shanghai Pulmonary Hospital, School of Medicine, Tongji University, Shanghai, China).

Comment on: Aly RG, Rekhtman N, Li X, et al. Spread Through Air Spaces (STAS) Is Prognostic in Atypical Carcinoid, Large Cell Neuroendocrine Carcinoma, and Small Cell Carcinoma of the Lung. J Thorac Oncol 2019;14:1583-93.

Submitted Sep 24, 2019. Accepted for publication Oct 22, 2019.

doi: $10.21037 /$ tlcr.2019.10.20

View this article at: http://dx.doi.org/10.21037/tlcr.2019.10.20

Spread through air spaces (STAS) was proposed as a new form of cancer invasion by the World Health Organization in 2015. Since the definition of STAS, a number of studies on STAS have been reported and revealed the clinical significance of STAS in various histological subtypes of lung cancer.

The recent report by Aly et al., "Tumor Spread Through Air Spaces (STAS) is Prognostic in Atypical Carcinoid, Large Cell Neuroendocrine Carcinoma, and Small Cell Carcinoma of Lung," was published in the Fournal of Thoracic Oncology (1). While STAS has been reported as a prognostic factor of lung adenocarcinoma, squamous cell carcinoma and pleomorphic carcinoma in previous reports, few studies have evaluated the clinical significance of STAS in lung neuroendocrine tumors (NETs) (2-4). In this retrospective analysis, the authors investigated the correlation between STAS and the prognosis in patients with resected lung NETs, including typical carcinoid (TC), atypical carcinoid (AC), large cell neuroendocrine carcinoma (LCNEC) and small cell carcinoma (SCLC). They concluded that STAS was significantly associated with early distant metastasis and cancer-specific death. In addition, they found that STAS was an independent prognosticator in patients with LCNEC and SCLC. In brief, the authors investigated 487 patients with lung NETs (299 with TC, 38 with AC, 93 with LCNEC and 57 with SCLC), and a higher incidence of STAS was detected in histologic subtypes with a higher grade than with a lower grade $[16 \%$ of TCs, $37 \%$ of ACs, $43 \%$ of LCNECs, and $46 \%$ of SCLCs $(\mathrm{P}<0.001)]$. The presence of STAS was associated with following clinicopathological features, smoking history; adjuvant therapy; lymph node metastasis; higher stage; lymphatic, vascular, and pleural invasion; higher percentage of necrosis; higher mitotic count; and higher Ki-67 index, compared with STAS-negative tumors. STAS was also associated with an increased risk of distant metastasis, especially brain metastasis (3-fold higher risk than patients without STAS) and an increased cumulative incidence of recurrence [sub-hazard ratio (SHR) $=2.85,95 \%$ confidence interval $(\mathrm{CI}): 1.73-4.68, \mathrm{P}<0.001)$ and cancerspecific cumulative death ( $\mathrm{SHR}=2.72,95 \%$ CI: $1.57-4.70$, $\mathrm{P}<0.001)$. The results appear to be clinically meaningful, as a limited number of lung NETs, especially SCLC, undergo surgical resection.

The clinical significance of STAS was first established in adenocarcinoma and subsequently reported to be a prognostic factor in squamous cell carcinoma and pleomorphic carcinoma. STAS is defined as the presence of one or more pathologic micropapillary clusters, solid nests or single cells beyond the edge of the tumor that move into the air spaces in the surrounding lung parenchyma, and further classifications of STAS and exclusion criteria have been adopted in studies (5). Since few cases of small cell lung cancer are resected, there are few reports about the significance of STAS in lung NETs. Our previous report evaluating the clinical significance of STAS in SCLC 
found that STAS was detected in about $80 \%$ of SCLC patients, and there was no association between STAS and the prognosis (6). The differences between the report by Aly et al. and our previous report might have been due to differences in the definition of STAS or the number of patients examined.

In the present report, STAS was associated with distant metastasis, especially brain metastasis, and this is the first report to reveal a relationship between STAS and brain metastasis. Several previous reports evaluated the relationship between STAS and the recurrence pattern. A report by Yanagawa et al. evaluating the association between STAS and the recurrence pattern in patients with stage I-III lung squamous cell carcinoma found that STAS positivity was associated with frequent events of local recurrence in all stages (7). In stage I patients, the presence of STAS was related to both local and distant recurrence, while STAS was associated with only local recurrence in stage II and III patients. In addition, Kadota et al. found that STAS was correlated with an increased risk of both distant and loco-regional recurrence in patients with stage I lung adenocarcinoma undergoing limited resection (2).

However, it has been reported that differences in surgical procedures might influence the clinical significance of STAS and the recurrence pattern in STAS-positive patients. Eguchi et al. reported that patients with STAS-positive T1 lung adenocarcinoma who underwent sublobar resection more frequently developed locoregional recurrence than patients with lobectomy (8). Shiono et al. reported that the presence of STAS was a prognostic factor in patients who underwent sublobar resection, but not in the lobectomy group (9). They also revealed that sublobar resection for patients with STAS resulted in a greater frequency of pulmonary metastasis than in patients who underwent lobectomy. These results suggest that the surgical margin might be deeply involved in the higher frequency of local recurrence in STAS-positive patients than in STAS-negative ones.

To exclude the influence of surgical procedure, Terada et al. evaluated patients with pathological stage III (N2) lung adenocarcinoma who underwent lobectomy or pneumonectomy with systematic node dissection (10). They found that the presence of STAS was a predictor of distant recurrence but not local recurrence. In our previous report evaluating the prognostic significance of STAS in lung adenocarcinoma with lymph node metastasis, none of the patients underwent sublobar resection, and there was no marked difference in the recurrence pattern according to the STAS status (11).
Previous reports evaluating the relationship between STAS and clinicopathological features showed that the presence of STAS was also associated with lymphovascular invasion, which may be associated with nodal or distant metastasis. However, in the present report, there was no correlation between STAS and these factors in the AC, LCNEC or SCLC subsets, although this absence of a correlation might have been due to the small number of cases involved. It therefore seems difficult to definitively explain the relationship between the recurrence pattern and the presence of STAS.

In the present report, STAS was associated with early recurrence after surgery and distant metastasis, suggesting that systemic treatment might be required in addition to local treatment by surgery in STAS-positive cases. Prophylactic cranial irradiation (PCI) is recommended to improve both the overall and disease-free survival in patients with limiteddisease (LD)-SCLC after the achievement of complete remission through chemoradiotherapy (12). Surgical resection is reportedly an effective treatment for stage I LDSCLC patients as well as some stage II and III patients, and the combination of surgery and chemotherapy is often performed in resectable early SCLC $(13,14)$. If STAS is a risk factor of brain metastasis, as reported in the present study, PCI might further improve the prognosis in resected early-stage SCLC with STAS, although this will need to be confirmed in a prospective study in the future. Similarly, the presence of STAS might be an indication for the introduction of postoperative adjuvant chemotherapy in NSCLC.

Although the clinical significance of STAS has been reported in various studies, the biological significance of STAS has not been fully clarified. STAS has been reported to be associated with Kirsten rat sarcoma viral oncogene homolog mutations, epidermal growth factor receptor wild-type and anaplastic lymphoma kinase fusions in lung adenocarcinomas $(2,15,16)$; however, whether or not this association is applicable to other histologies is unclear. The HGF/MET pathway is known to be involved in the acquisition of an invasive phenotype in NSCLC, and Lapere et al. reported elevated levels of MET and phosphoMET at the invasion front of the tumor and in tumor cells spreading through air spaces in lung adenocarcinoma (17). Jin et al. reported that c-ros oncogene 1-rearranged lung cancer was associated with frequent aerogenous spread tumor cells with decreased E-cadherin expression, while Yanagawa $e t$ al. found that the expression of p53 and E-cadherin was not associated with the status of STAS $(7,18)$. However, the in vivo effect of STAS remains unclear, so 
several studies have suggested that STAS might simply be "loose tumor fragments" or artifacts caused by spreading through a knife surface (19). The driver oncogene status, biological features and clinical behavior differ according to the histological type. If STAS is really related to the patients' prognosis, regardless of the histological type and other clinicopathological factor, it should be considered a new form of cancer invasion and treated like any other pathological factor, such as lymphovascular and pleural invasion.

In conclusion, the clinical significance of STAS has been increasing in all histological types of lung cancer. However, several concerns about STAS remain to be addressed, including the biological significance and distinction from artifacts, and STAS still has its skeptics concerning whether or not it actually is a form of invasion. Further studies will be required in order to clarify the clinical significance of STAS, such as its reflection in the TNM classification or influence on the administration of adjuvant therapy.

\section{Acknowledgments}

We would like to thank Brian Quinn for his editing work on the manuscript.

\section{Footnote}

Conflicts of Interest: The authors have no conflicts of interest to declare.

Ethical Statement: The authors are accountable for all aspects of the work in ensuring that questions related to the accuracy or integrity of any part of the work are appropriately investigated and resolved.

\section{References}

1. Aly RG, Rekhtman N, Li X, et al. Spread Through Air Spaces (STAS) Is Prognostic in Atypical Carcinoid, Large Cell Neuroendocrine Carcinoma, and Small Cell Carcinoma of the Lung. J Thorac Oncol 2019;14:1583-93.

2. Kadota K, Nitadori J, Sima CS, et al. Tumor Spread through Air Spaces is an Important Pattern of Invasion and Impacts the Frequency and Location of Recurrences after Limited Resection for Small Stage I Lung Adenocarcinomas. J Thorac Oncol 2015;10:806-14.

3. Warth A, Muley T, Kossakowski CA, et al. Prognostic Impact of Intra-alveolar Tumor Spread in Pulmonary
Adenocarcinoma. Am J Surg Pathol 2015;39:793-801.

4. Lu S, Tan KS, Kadota K, et al. Spread through Air Spaces (STAS) Is an Independent Predictor of Recurrence and Lung Cancer-Specific Death in Squamous Cell Carcinoma. J Thorac Oncol 2017;12:223-34.

5. Travis WD, Brambilla E, Nicholson AG, et al. The 2015 World Health Organization Classification of Lung Tumors: Impact of Genetic, Clinical and Radiologic Advances Since the 2004 Classification. J Thorac Oncol 2015;10:1243-60.

6. Toyokawa G, Yamada Y, Tagawa T, et al. High Frequency of Spread Through Air Spaces in Resected Small Cell Lung Cancer. Anticancer Res 2018;38:1821-5.

7. Yanagawa N, Shiono S, Endo M, et al. Tumor spread through air spaces is a useful predictor of recurrence and prognosis in stage I lung squamous cell carcinoma, but not in stage II and III. Lung Cancer 2018;120:14-21.

8. Eguchi T, Kameda K, Lu S, et al. Lobectomy Is Associated with Better Outcomes than Sublobar Resection in Spread through Air Spaces (STAS)-Positive T1 Lung Adenocarcinoma: A Propensity Score-Matched Analysis. J Thorac Oncol 2019;14:87-98.

9. Shiono S, Endo M, Suzuki K, et al. Spread Through Air Spaces is a Prognostic Factor in Sublobar Resection of Non-Small Cell Lung Cancer. Ann Thorac Surg 2018;106:354-60.

10. Terada Y, Takahashi T, Morita S, et al. Spread through air spaces is an independent predictor of recurrence in stage III (N2) lung adenocarcinoma. Interact Cardiovasc Thorac Surg 2019;29:442-8.

11. Toyokawa G, Yamada Y, Tagawa T, et al. Significance of Spread Through Air Spaces in Resected Lung Adenocarcinomas With Lymph Node Metastasis. Clin Lung Cancer 2018;19:395-400.e1.

12. Aupérin A, Arriagada R, Pignon JP, et al. Prophylactic cranial irradiation for patients with small-cell lung cancer in complete remission. Prophylactic Cranial Irradiation Overview Collaborative Group. N Engl J Med 1999;341:476-84.

13. Takei H, Kondo H, Miyaoka E, et al. Surgery for small cell lung cancer: a retrospective analysis of 243 patients from Japanese Lung Cancer Registry in 2004. J Thorac Oncol 2014;9:1140-5.

14. Takenaka T, Takenoyama M, Inamasu E, et al. Role of surgical resection for patients with limited disease-small cell lung cancer. Lung Cancer 2015;88:52-6.

15. Onozato ML, Kovach AE, Yeap BY, et al. Tumor islands in resected early-stage lung adenocarcinomas are associated 
with unique clinicopathologic and molecular characteristics and worse prognosis. Am J Surg Pathol 2013;37:287-94.

16. Lee JS, Kim EK, Kim M, et al. Genetic and clinicopathologic characteristics of lung adenocarcinoma with tumor spread through air spaces. Lung Cancer 2018;123:121-6.

17. Lapère $\mathrm{C}$, Cortot $\mathrm{AB}$, Gregoire $\mathrm{V}$, et al. Preferential Localization of MET Expression at the Invasion Front and in Spreading Cells Through Air Spaces in Non-Small Cell
Lung Carcinomas. Am J Surg Pathol 2017;41:414-22.

18. Jin Y, Sun PL, Park SY, et al. Frequent aerogenous spread with decreased E-cadherin expression of ROS1-rearranged lung cancer predicts poor disease-free survival. Lung Cancer 2015;89:343-9.

19. Blaauwgeers H, Russell PA, Jones KD, et al. Pulmonary loose tumor tissue fragments and spread through air spaces (STAS): Invasive pattern or artifact? A critical review. Lung Cancer 2018;123:107-11.
Cite this article as: Kozuma Y, Toyokawa G, Yamada Y, Shoji F, Yamazaki K, Oda Y, Takeo S. Spread through air spaces in lung neuroendocrine tumor. Transl Lung Cancer Res 2019;8(Suppl 4):S439-S442. doi: 10.21037/tlcr.2019.10.20 